\title{
P033. Headache and commuting: preliminary data in a group of workers
}

\author{
Ennio Pucci ${ }^{1 *}$, Giuseppe Taino ${ }^{2}$, Marcello Imbriani ${ }^{3}$, Alfredo Costa ${ }^{1}$, Silvano Cristina ${ }^{1}$, Fabio Antonaci ${ }^{1}$ \\ From Abstracts from the 1st Joint ANIRCEF-SISC Congress \\ Rome, Italy. 29-31 October 2015
}

\section{Introduction}

The different forms of primary headache disorders are highly prevalent which, taken together, affect a greater extent of people of working age. Risk factors of headache triggers can be physical, psycho-social or organizational (work in shifts, night work, working conditions non-ergonomic, etc.). Commuting is a phenomenon which consists in the double daily shift of people moving, usually by public transport.

\section{Objective}

The aim of this study was to study the phenomenon of commuting, especially the prevalence, in a group of workers of a chemical industry.

\section{Patients and methods}

Health surveillance and medical history questionnaires focused on employment and on the presence of primary headache in 95 workers of a chemical industry $(91 \mathrm{M}$, 4 F). Night shift work interested $52.6 \%$ of workers, while $47.4 \%$ worked during the day. The diagnosis of migraine was defined according to the criteria of the ICHD-III beta version.

\section{Results}

The analysis of the questionnaires and the processing of the results showed that the form of primary headache with higher prevalence, in both night shift workers and day workers, was represented by migraine without aura (51.5\% of all headache workers) followed by tension-type headache $(42.5 \%)$ and by migraine with aura (6\%). The different prevalence of primary headaches in the two groups of workers (shift and day workers) did not reach statistical significance. We then decided to consider instead the commuting variable since $46 \%$ of night shift workers were also commuters. Processing of the data of the subgroup showed a statistically significant association between the prevalence of primary headache and commuting/night shift $(\mathrm{p}<0.05)$.

\section{Conclusions}

The commuters/shift workers are more prone to develop a primary headache, especially migraine without aura. Further investigations are needed to better clarify the association between primary headache and commuting/shift working, and also, more generally, "unusual" rhythm of work.

Written informed consent to publication was obtained from the patient(s).

\section{Authors' details \\ ${ }^{1}$ Headache Science Center, University Consortium for the Study of Adaptive Disorders and Headache (UCADH), Department of Brain and Behavioral Sciences, University of Pavia, IRCCS "C. Mondino", Pavia, Italy. ${ }^{2}$ U.O. Hospital Occupational Medicine, Foundation IRCCS "S. Maugeri", Pavia, Italy. ${ }^{3}$ Department of Public Health, Experimental and Forensic Medicine, University of Pavia, Pavia, Italy.}

Published: 28 September 2015

doi:10.1186/1129-2377-16-S1-A79

Cite this article as: Pucci et al:: P033. Headache and commuting:

preliminary data in a group of workers. The Journal of Headache and Pain 2015 16(Suppl 1):A79.

\footnotetext{
* Correspondence: ennio.pucci@mondino.it

${ }^{1}$ Headache Science Center, University Consortium for the Study of Adaptive Disorders and Headache (UCADH), Department of Brain and Behavioral

Sciences, University of Pavia, IRCCS "C. Mondino", Pavia, Italy

Full list of author information is available at the end of the article
} 